\title{
An approximate analytical solution of Richards equation with finite boundary
}

\section{Xi Chen ${ }^{1}$ and Ying Dai ${ }^{*}$}

\section{*Correspondence:}

1291415113@qq.com

25chool of Aerospace Engineering

and Applied Mechanicas, Tongji

University, Shanghai, China

Full list of author information is

available at the end of the article

\begin{abstract}
We apply a series expansion technique to estimate the water content distribution and front position in finite boundary conditions. We derive an approximate analytical solution of the Richards equation (RE) for the horizontal infiltration problem. The solution is suitable for arbitrary hydraulic diffusivity in water infiltration. Compared with the finite element method, two examples in power law diffusivity and van Genuchten model are shown to test the accuracy of present approximation.
\end{abstract}

MSC: 35C10; 35K20; 76505

Keywords: series expansion technique; finite boundary; approximate analytical solution; Richards equation; van Genuchten model

\section{Introduction}

The Richards equation (RE) presents the movement of water in unsaturated soils [1]. It is usually a nonlinear parabolic partial differential equation for one-dimensional horizontal infiltration problem, which can be expressed as $[2,3]$

$$
\frac{\partial \theta}{\partial t}=\frac{\partial}{\partial x}\left(D(\theta) \frac{\partial \theta}{\partial x}\right)
$$

where $\theta$ is the volumetric water content of unsaturated porous media, $x$ and $t$ are the space and time coordinates, and $D(\theta)$ is the hydraulic diffusivity. We consider the analytical solution in a finite domain $0 \leq x \leq L$, which owns the Dirichlet boundary conditions

$$
\left.\theta\right|_{x=0}=\theta_{L},\left.\quad \theta\right|_{x=L}=\theta_{0},
$$

where $\theta_{L}\left(\neq \theta_{0}\right)$ and $\theta_{0}$ are constant moisture contents in wetting front analysis, and the initial condition is

$$
\left.\theta\right|_{t=0}=\theta_{0}
$$

In these years, Heaslet and Alksne technique [4], perturbation technique [5], traveling wave method [6], and series method [7-10] are used to obtain an analytical solution of RE with semi-infinite boundaries. Fourier transformation, separation of variables [11, 12], and

(c) The Author(s) 2017. This article is distributed under the terms of the Creative Commons Attribution 4.0 International License (http://creativecommons.org/licenses/by/4.0/), which permits unrestricted use, distribution, and reproduction in any medium, provided you give appropriate credit to the original author(s) and the source, provide a link to the Creative Commons license, and indicate if changes were made. 
other approaches [13-19] are applied for some linear or linearized RE with finite boundaries (2). Nevertheless, there are few approximate analytical solutions of equations (1)-(3) that can illustrate the long-time behavior for water infiltration in constant moisture content boundary conditions of equation (2), especially when the hydraulic diffusivity is an arbitrary function of $\theta$. Our goal is deriving an approximate analytical solution of equations (1)-(3).

Motivated by the methods and approaches mentioned, we focus on analyzing the water infiltration varying from a transient state to steady one and try to approximate the changes of water profile. By a series expansion technique we construct an approximate solution about space variable $x$ and water profile to approximate the water content distribution for arbitrary diffusivity in RE. In addition, we analyze the relationship of time variable $t$ and $\theta$ in a definite space coordinate.

\section{Wetting front analysis}

The RE for horizontal infiltration with initial and boundary conditions equations (2)-(3) in this paper is divided into two parts: water infiltration in semiinfinite layer and in finite layer by boundary analysis $[20,21]$. Before the wetting front arrives at the boundary point $x=L$, it is considered as a semiinfinite boundary problem, which is well approximated by the Boltzmann solution [4], shifted front solution [5], and series solution [9]. The profiles of infiltration in semiinfinite layer are shown by dotted lines in Figure 1, and the front position of the finite boundary problem is shown by solid and thick solid lines in Figure 1. The thick solid line shows a steady flow for water infiltration and presents a steady state that is irrelevant to time variable $t$. The steady flow can be described by the ordinary differential equation

$$
\frac{\mathrm{d}}{\mathrm{d} x}\left(D(\theta) \frac{\mathrm{d} \theta}{\mathrm{d} x}\right)=0
$$

with Dirichlet boundary conditions

$$
\left.\theta\right|_{x=0}=\theta_{L},\left.\quad \theta\right|_{x=L}=\theta_{0} .
$$

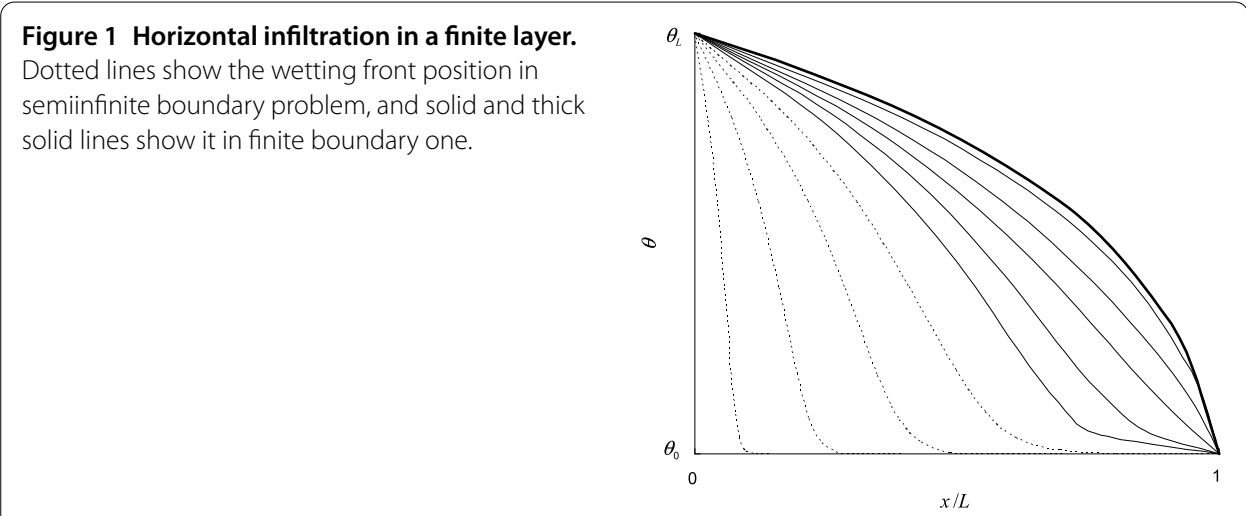


The analytical solution of equation (4) with (5) can be solved as

$$
x=\frac{L}{\int_{\theta_{0}}^{\theta_{L}} D \mathrm{~d} \theta}\left(\int_{\theta_{0}}^{\theta_{L}} D \mathrm{~d} \theta-\int_{\theta_{0}}^{\theta} D \mathrm{~d} \theta\right) .
$$

The solid lines in Figure 1 show the process of water infiltration varying from a transient flow to steady one. In this section, we construct an approximate analytical solution to simulate the infiltrate process.

Introducing the Boltzmann variable $\phi=x / t^{1 / 2}$, we can transform equation (1) as

$$
-\frac{1}{2} \phi \frac{\mathrm{d} \theta_{r}}{\mathrm{~d} \phi}=\frac{\mathrm{d}}{\mathrm{d} \phi}\left(D\left(\theta_{r}+\theta_{0}\right) \frac{\mathrm{d} \theta_{r}}{\mathrm{~d} \phi}\right)
$$

where $\theta_{r}=\theta-\theta_{0}$. Integrating equation (7), we obtain

$$
\int_{0}^{\theta_{r}} \phi \mathrm{d} \theta_{r}=-2 D\left(\theta_{r}+\theta_{0}\right) \frac{\mathrm{d} \theta_{r}}{\mathrm{~d} \phi}+C
$$

where

$$
C=\left.2 D\left(\theta_{r}+\theta_{0}\right) \frac{\mathrm{d} \theta_{r}}{\mathrm{~d} \phi}\right|_{\theta_{r}=0} .
$$

By the mean value theorem equation (8) can be written as

$$
\phi\left(\eta \theta_{r}\right)=-2 \frac{D\left(\theta_{r}+\theta_{0}\right)}{\theta_{r}} \frac{\mathrm{d} \theta_{r}}{\mathrm{~d} \phi}+\frac{C}{\theta_{r}},
$$

where $\eta \in(0,1)$. Substituting $\theta_{\beta}=\eta \theta_{r}$ and $\theta_{r}=\theta_{\beta}+a$ into equation (10), we obtain

$$
\phi\left(\theta_{\beta}\right)=-2 \frac{D\left(\theta_{\beta}+a+\theta_{0}\right)}{\theta_{\beta}} \frac{\mathrm{d} \theta_{\beta}}{\mathrm{d} \phi}+\frac{C}{\theta_{\beta}+a},
$$

where $a$ is a constant. Integrating equation (11), we obtain

$$
\int_{0}^{\phi}\left[\phi\left(\eta\left(\theta_{\beta}+a\right)+\theta_{0}\right)-\frac{C}{\theta_{\beta}+a}\right] \mathrm{d} \phi=2 \int_{\theta_{\beta}}^{\theta_{L}-\theta_{0}-a} \frac{D\left(\theta_{\beta}+a+\theta_{0}\right)}{\theta_{\beta}} \mathrm{d} \theta_{\beta},
$$

which yields

$$
\left[\phi\left(\eta\left(\theta_{\zeta}+a\right)+\theta_{0}\right)-\frac{C}{\theta_{\zeta}+a}\right] \phi=2 \int_{\theta_{\beta}}^{\theta_{L}-\theta_{0}-a} \frac{D\left(\theta_{\beta}+a+\theta_{0}\right)}{\theta_{\beta}} \mathrm{d} \theta_{\beta},
$$

where $\theta_{\zeta}=\theta_{\beta}+\zeta\left(\theta_{L}-\theta_{0}-a-\theta_{\beta}\right)$ and $\zeta \in(0,1)$. Equation (13) shows the relationship between the Boltzmann variable $\phi$ and the integration function $\xi$,

$$
\xi=\int_{\theta_{\beta}}^{\theta_{L}-\theta_{0}-a} \frac{D\left(\theta_{\beta}+a+\theta_{0}\right)}{\theta_{\beta}} \mathrm{d} \theta_{\beta}
$$


Influenced by a series expansion technique [9], we show the analytical solution of the Boltzmann variable as a series

$$
\phi(\xi) \approx \sum_{i=1}^{n} U_{i} \xi^{i}
$$

where $U_{i}(i=1,2,3, \ldots, n)$ are calculation parameters. Based on zero and $\theta_{\beta}$, we apply the integer-order derivatives theorem [22] to equation (8):

$$
\left.\frac{\mathrm{d}^{(k)}}{\mathrm{d} \theta_{\beta}^{(k)}}\left(\int_{0}^{\theta_{\beta}} \phi \mathrm{d} \theta_{\beta}+2 D \frac{\mathrm{d} \theta_{\beta}}{\mathrm{d} \phi}\right)\right|_{\theta_{\beta}=\theta_{L}-\theta_{0}-a}=\left.2 \frac{\mathrm{d}^{(k)}}{\mathrm{d} \theta_{\beta}^{(k)}}\left[\left.D \frac{\mathrm{d} \theta_{\beta}}{\mathrm{d} \phi}\right|_{\theta_{\beta}=0}\right]\right|_{\theta_{\beta}=\theta_{L}-\theta_{0}-a},
$$

where $k=0,1,2,3, \ldots, n-1$. The parameters $U_{i}$ can be obtained by substituting equation (15) into equation (16) and solving nonlinear algebraic equations. When $a=0$, equation (15) with (16) is the series solution for semiinfinite problem [9]. Considering this, we construct an approximate relationship between space variable $x$ and water content $\theta_{\beta}$ in both semiinfinite and finite boundaries as

$$
x \approx \begin{cases}\sqrt{t} \phi, & a=0, t \leq t_{L} \\ \frac{L}{\left.\phi\right|_{\theta_{\beta}=0}} \phi, & 0>a \geq a_{L}, t>t_{L}\end{cases}
$$

where $a_{L}$ can be solved by comparing equation (17) and Taylor expansion of equation (6) based on $\xi, t_{L}$ is the time of the wetting front that arrives at the finite boundary point $x=L$ and can be calculated by substituting $x=L$ into equation (17):

$$
t_{L} \approx L^{2} /\left(\sum_{i=1}^{n} U_{i}\left(\int_{0}^{\theta_{L}-\theta_{0}} \frac{D}{\widetilde{\theta_{\beta}}} \mathrm{d} \widetilde{\theta_{\beta}}\right)^{i}\right)^{2} .
$$

In equation (17), the approximations of water profiles for horizontal infiltration are shown as the calculation parameter $a$ varies. When $t>t_{L}$, we substitute $\theta_{r}=\theta-\theta_{0}, \theta_{r}=\theta_{\beta}+a$, and equation (17) into equation (1):

$$
\frac{\partial \theta}{\partial t}=\frac{\left.\phi\right|_{\theta_{\beta}=0}}{L}\left(\frac{\partial \phi}{\partial \theta_{\beta}}\right)^{-1}+\frac{\partial}{\partial \theta_{\beta}}\left(D \frac{\left.\phi\right|_{\theta_{\beta}=0}}{L}\left(\frac{\partial \phi}{\partial \theta_{\beta}}\right)^{-1}\right) .
$$

Substituting equations (14) and (15) into (17), $\theta_{\beta}$ can be shown as a function of $a$ in a definite space point $x=x_{d} \in(0, L)$ :

$$
x_{d} \approx \frac{L \sum_{i=1}^{n} U_{i}\left(\int_{\theta_{\beta}}^{\theta_{L}-\theta_{0}-a} \frac{D}{\theta_{\beta}} \mathrm{d} \tilde{\theta}_{\beta}\right)^{i}}{\sum_{i=1}^{n} U_{i}\left(\int_{0}^{\theta_{L}-\theta_{0}-a} \frac{D}{\theta_{\beta}} \mathrm{d} \tilde{\theta_{\beta}}\right)^{i}},
$$

and $\theta=\theta_{0}+\theta_{\beta}+a$. We take $a$ as

$$
a=a_{j} \quad(j=0,1,2,3, \ldots)
$$

where $a_{j} \in\left[a_{L}, 0\right)$ is a given constant. Then the corresponding $\theta_{j}$ can be obtained from equation (20). Substituting $a=a_{j}$ and $\theta_{j}=\theta_{\beta j}+\theta_{0}+a_{j}$ into equation (19), we can calculate 
the value of $\partial \theta / \partial t$ at $x=x_{d}$. We apply a polynomial series to approximate the changes

$$
\frac{\partial \theta}{\partial t} \approx \sum_{k=0}^{n_{1}} V_{k} \theta^{k}
$$

where $k=0,1,2,3, \ldots, n_{1}$, and $V_{k}$ can be calculated by the least-squares method. Then the time variable $t$ can be solved:

$$
t=\int_{\theta_{\beta 0}}^{\theta} \frac{1}{\sum_{k=0}^{n_{1}} V_{k} \theta^{k}} \mathrm{~d} \theta-t_{L}
$$

where $\theta_{\beta 0}$ is the water content as the wetting front arriving at the boundary point $x=L$ and can be solved from

$$
\frac{x_{d}}{\sqrt{t_{L}}} \approx \sum_{i=1}^{n} U_{i}\left(\int_{\theta_{\beta 0}}^{\theta_{L}-\theta_{0}} \frac{D}{\widetilde{\theta}_{\beta}} \mathrm{d} \tilde{\theta}_{\beta}\right)^{i} .
$$

\section{Numerical simulation}

In this section, two examples are shown to confirm the accuracy of the present method.

Example 1 (Power-law model) We take the power law diffusivity from Hall's mortar [23, 24] model as $D(\theta)=247.1 \theta^{4}$. The initial and boundary values are taken as $\theta_{0}=0.5$ and $\theta_{L}=1$, respectively, and $L$ in equation (2) is $13 \mathrm{~mm}$. Before the wetting front arrives at the point $x=13 \mathrm{~mm}$, it can be treated as a semiinfinite problem. Applying equation (17) at $a=0$, we have

$$
\phi \approx \sum_{i=1}^{n} U_{i}\left(\int_{\theta_{r}}^{0.5} \frac{247.1\left(\theta_{r}+0.5\right)^{4}}{\theta_{r}} \mathrm{~d} \theta_{r}\right)^{i},
$$

where $U_{i}$ are calculated as $n$ varies from 1 to 5 .

$$
\begin{aligned}
& n=1: U_{1}=0.1445249313 \\
& n=2: U_{1}=0.1667699993, U_{2}=-3.374544704 \times 10^{-4} . \\
& n=3: U_{1}=0.1571011460, U_{2}=-3.178898137 \times 10^{-4}, U_{3}=8.78800083 \times 10^{-7} . \\
& n=4: U_{1}=0.1620248146, U_{2}=-3.278527209 \times 10^{-4}, U_{3}=9.921998925 \times 10^{-7}, \\
& U_{4}=-3.797520062 \times 10^{-9} . \\
& n=5: U_{1}=0.1589918636, U_{2}=-3.217156285 \times 10^{-4}, U_{3}=9.214216339 \times 10^{-7}, \\
& U_{4}=-3.620798276 \times 10^{-9}, U_{5}=1.360893606 \times 10^{-11} .
\end{aligned}
$$

Compared with the finite element method (FEM), we depict approximations of orders 1-5 of $\phi$ in Figure 2 and present relative errors of the 5th-order solution in Table 1.

We obtain that the present approximations are more close to the FEM as order increases from 1 to 5 in Figure 2, and the maximum relative error is $1.353 \%$ in $20.1946 \mathrm{~mm} / \mathrm{min}^{1 / 2}$ in Table 1. According to the fith-order approximation above and equation (18), we obtain that $t_{L}=0.1276 \mathrm{~min}$ when the wetting front arrives at the boundary point $x=13 \mathrm{~mm}$. Applying equations (19)-(24), $x_{d}=9 \mathrm{~mm}$ is taken as a definite space value in $a_{j}$ and $\theta_{j}$ calculation, and the sixth-order approximation of $\partial \theta / \partial t$ is presented as

$$
\begin{aligned}
\frac{\partial \theta}{\partial t} \approx & -13,667.969585 \theta^{6}+56,224.381941 \theta^{5}-96,658.819517 \theta^{4}+88,993.549536 \theta^{3} \\
& -46,337.629110 \theta^{2}+12,947.589875 \theta-1,515.864672
\end{aligned}
$$


Figure 2 Results of $\phi$ obtained from the FEM and approximations of orders 1-5.

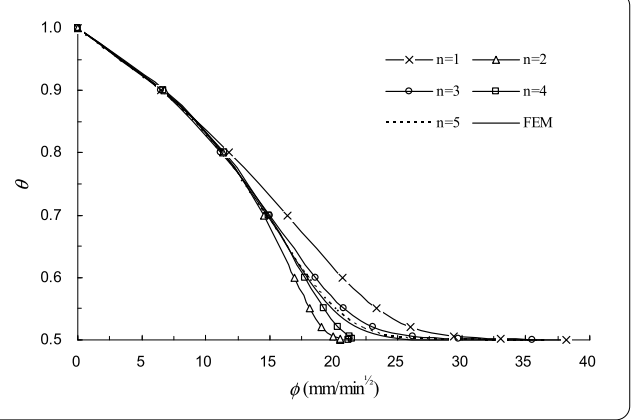

Table 1 Results of $\theta$ obtained from the FEM and fifth-order approximation

\begin{tabular}{llll}
\hline $\boldsymbol{\phi}\left(\mathbf{m m} / \mathbf{m i n}^{\mathbf{1 / 2}}\right)$ & Present method & FEM & Relative error (\%) \\
\hline 36.3899 & 0.5001 & 0.5 & 0.02 \\
20.1946 & 0.55 & 0.54266 & 1.353 \\
18.1551 & 0.6 & 0.594696 & 0.892 \\
14.8670 & 0.7 & 0.69924 & 0.109 \\
11.2396 & 0.8 & 0.8007 & -0.087 \\
6.5490 & 0.9 & 0.900045 & -0.005 \\
0 & 1 & 1 & 0 \\
\hline
\end{tabular}

Figure 3 The changes of water profile for power law model. Dotted lines show the present approximations, and solid lines show the results by the FEM.

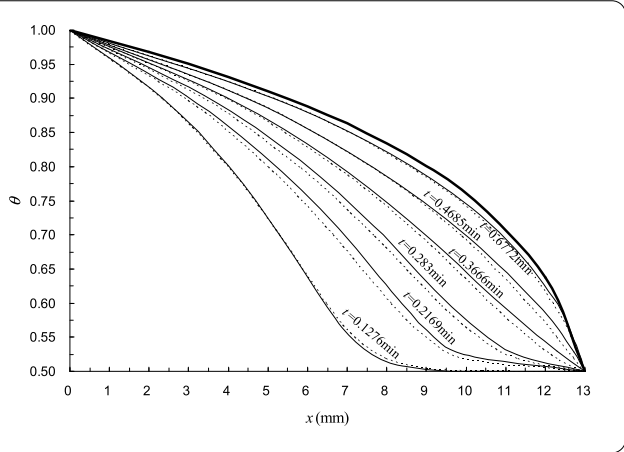

When $t \geq t_{L}$, the fifth-order approximation between the space variable $x$ and water content $\theta$ is

$$
\begin{aligned}
x \approx & \frac{13}{\sum_{i=1}^{5} U_{i}\left(\int_{0}^{0.5-a} \frac{247.1\left(\theta_{\beta}+a+0.5\right)^{4}}{\theta_{\beta}} \mathrm{d} \theta_{\beta}\right)^{i}} \\
& \times \sum_{i=1}^{5} U_{i}\left(\int_{\theta-a-0.5}^{0.5-a} \frac{247.1\left(\theta_{\beta}+a+0.5\right)^{4}}{\theta_{\beta}} \mathrm{d} \theta_{\beta}\right)^{i},
\end{aligned}
$$

where $U_{i}$ can be obtained by solving equation (16) for different $a$. As $t$ increases, we calculate the approximations for $a=0, a=-0.001, a=-0.005, a=-0.025, a=-0.1$, and $a=-0.2$, and the corresponding time by solving equation (26). The corresponding water profiles are shown in Figure 3, which presents the process of water infiltration varying from transient state to steady one. The thick solid line in Figure 3 is derived by equation (6) and shows the steady solution in front analysis. The solid lines and dotted ones are obtained by the FEM and present method, respectively, and show the changes of water profiles as time goes by. Compared with the FEM, the relative errors between solid lines and dotted ones are shown in Figure 4. 
Figure 4 Relative errors of present solutions and the FEM.

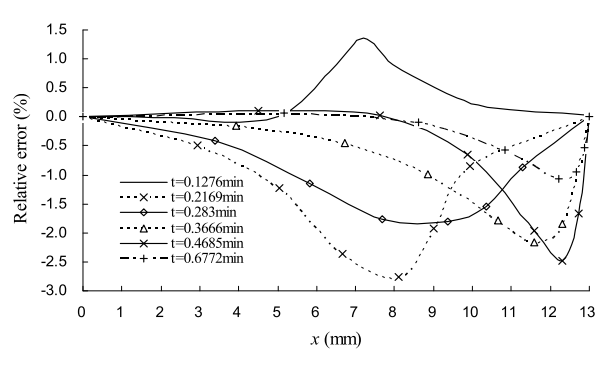

Figure 5 Relationship between $t$ and $\theta$ in $x_{d}=9 \mathrm{~mm}$.

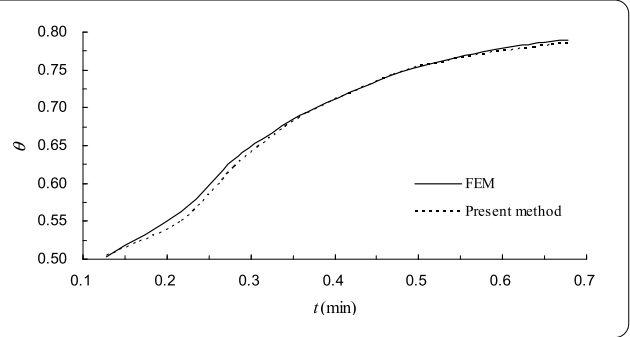

Table 2 The results of $\theta$ obtained from the present method and the FEM in $x_{d}=9 \mathrm{~mm}$

\begin{tabular}{lllc}
\hline $\boldsymbol{t}$ (min) & Present method & FEM & Relative error (\%) \\
\hline 0.1276 & 0.505193 & 0.5027 & 0.4959 \\
0.2169 & 0.550959 & 0.562178 & -1.9956 \\
0.2794 & 0.62067 & 0.6319 & -1.7772 \\
0.3149 & 0.65244 & 0.6587 & -0.9504 \\
0.3666 & 0.692165 & 0.69494 & -0.3993 \\
0.4685 & 0.742965 & 0.743 & -0.0047 \\
0.5337 & 0.76222 & 0.7638 & -0.2069 \\
0.5765 & 0.77126 & 0.7737 & -0.3154 \\
0.6353 & 0.780382 & 0.7841 & -0.4742 \\
0.658 & 0.783064 & 0.7874 & -0.5507 \\
0.6772 & 0.785114 & 0.7897 & -0.5807 \\
\hline
\end{tabular}

We observe that the present approximation is agreed with numerical solution, and the maximum relative error of present solution is less than $-3 \%$ in Figure 4 . In addition, we solve equation (26) and derive the relationship between the time variable $t$ and water content $\theta$, which shows that the present solution approximates well with the FEM as time increases in Figure 5, and the maximum relative error is less than 2\% in Table 2.

Example 2 (van Genuchten model) The hydraulic diffusivity defined by the van Genuchten model $[25,26]$ is

$$
D(\theta)=K_{s} k_{r} \frac{\partial H}{\partial \theta}
$$

where $K_{s}$ is the saturated hydraulic conductivity, $H$ is the water head, and $k_{r}$ is the intrinsic permeability [26]

$$
\begin{aligned}
& H=\frac{1}{\alpha}\left[\left(\frac{\theta-\theta_{R}}{\theta_{S}-\theta_{R}}\right)^{-1 / m}-1\right]^{1 / n}, \\
& k_{r}=\left(\frac{\theta-\theta_{R}}{\theta_{S}-\theta_{R}}\right)^{1 / 2}\left[1-\left(1-\left(\frac{\theta-\theta_{R}}{\theta_{S}-\theta_{R}}\right)^{1 / m}\right)^{m}\right]^{2},
\end{aligned}
$$


where $\theta_{R}$ is the residual volumetric water content, $\theta_{S}$ is the saturated volumetric water content, $\alpha$ is a parameter related to the mean pore-size, $n$ is a parameter related to the uniformity of the pore-size distribution, and $m=1-1 / n$. We take the parameters of Glendale clay loam [27] as $\theta_{R}=0.106, \theta_{S}=0.469, \alpha=1.04 \mathrm{~m}^{-1}, m=0.283$, and $K_{s}=1.52 \times 10^{-6} \mathrm{~m} \cdot \mathrm{s}^{-1}$. The length of $L$ is taken as $2 \times 10^{-3} \mathrm{~m}$, and the initial and boundary values in equations (2)-(3) are $\theta_{0}=0.25$ and $\theta_{L}=0.4$.

According to (28)-(29), the diffusivity in (1) is

$$
D\left(\theta_{r}+0.25\right)=\frac{c_{1}\left[1-\left(1-\left(c_{2}\left(\theta_{r}+0.25\right)-c_{3}\right)^{c_{4}}\right)^{0.283}\right]^{2}}{\left[\left(c_{2}\left(\theta_{r}+0.25\right)-c_{3}\right)^{-c_{4}}-1\right]^{c_{5}}\left(c_{2}\left(\theta_{r}+0.25\right)-c_{3}\right)^{c_{6}}},
$$

where $\theta_{r}=\theta-0.25$. Substituting the parameters of Glendale clay loam [27] into equations (28) and (29), we obtain that $c_{1}=0.1020084959 \times 10^{-4}, c_{2}=2.754820937, c_{3}=$ $0.2920110193, c_{4}=3.533568905, c_{5}=0.282999998$, in $c_{6}=4.033568905$ in equation (30). Similarly, we derive $\phi$ at $a=0$ before the wetting front travels through the boundary point $x=2 \times 10^{-3} \mathrm{~m}$ :

$$
\phi \approx \sum_{i=1}^{n} U_{i}\left(\int_{\theta_{r}}^{0.15} \frac{D\left(\theta_{r}+0.25\right)}{\theta_{r}} \mathrm{~d} \theta_{r}\right)^{i},
$$

where first- and second-order approximations of $U_{i}$ are

$$
\begin{aligned}
& n=1: U_{1}=3,266.070514 . \\
& n=2: U_{1}=3,600.557734, U_{2}=-2.796118274 \times 10^{9} .
\end{aligned}
$$

Compared with the FEM, first- and second-order approximate solutions of $\phi$ are presented in Figure 6, and the relative errors are shown in Table 3.

We observe that the present approximations are closer to the FEM as order varies from 1 to 2 in Figure 6, and the maximal value of the relative error is $-2.097 \%$ in 0.8397 $\mathrm{mm} / \mathrm{min}^{1 / 2}$ for second-order approximation in Table 3.

Figure 6 Results of $\phi$ obtained from the FEM and approximations of orders 1-2.

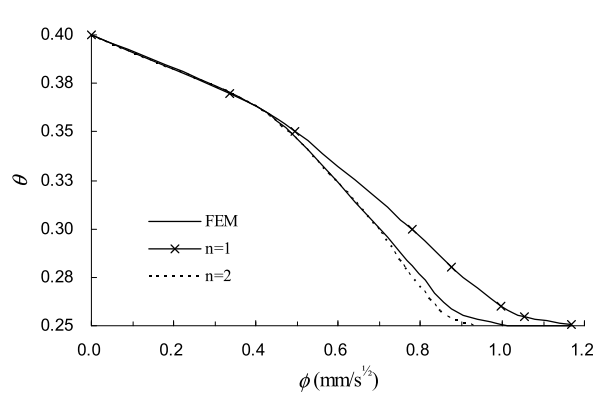

Table 3 Results of $\boldsymbol{\theta}$ obtained from the FEM and second-order approximation

\begin{tabular}{llll}
\hline $\boldsymbol{\phi}\left(\mathbf{m m} / \mathbf{s}^{\mathbf{1 / 2}}\right)$ & 2nd-order approximation & FEM & Relative error (\%) \\
\hline 0.9316 & 0.251 & 0.2529 & -0.757 \\
0.8717 & 0.255 & 0.2594 & -1.71 \\
0.8397 & 0.26 & 0.2656 & -2.097 \\
0.7651 & 0.28 & 0.2853 & -1.861 \\
0.7012 & 0.3 & 0.3033 & -1.076 \\
0.483 & 0.35 & 0.3499 & 0.025 \\
0.3418 & 0.37 & 0.3697 & 0.09 \\
0 & 0.4 & 0.4 & 0 \\
\hline
\end{tabular}


From the second-order approximation above and equation (18) we obtain $t_{L}=3.582 \mathrm{~s}$ when the wetting front arrives at the boundary point $x=2 \times 10^{-3} \mathrm{~m}$. Similarly, $x_{d}=$ $1.5 \times 10^{-3} \mathrm{~m}$ is taken as a definite space value in $a_{j}$ and $\theta_{j}$ calculation, and the sixth-order approximation of $\partial \theta / \partial t$ is presented as

$$
\begin{aligned}
\frac{\partial \theta}{\partial t} \approx & 5,672,855.52832 \theta^{6}-10,187,681.423 \theta^{5}+7,615,774.92597 \theta^{4} \\
& -3,033,314.6831 \theta^{3}+678,883.519123 \theta^{2}-80,948.6314005 \theta \\
& +4,017.3694807
\end{aligned}
$$

Then the second-order approximation between the space variable $x$ and water content $\theta$ is

$$
x \approx \frac{0.002}{\sum_{i=1}^{2} U_{i}\left(\int_{0}^{0.15-a} \frac{D\left(\theta_{\beta}+a+0.25\right)}{\theta_{\beta}} \mathrm{d} \theta_{\beta}\right)^{i}} \sum_{i=1}^{2} U_{i}\left(\int_{\theta-a-0.25}^{0.15-a} \frac{D\left(\theta_{\beta}+a+0.25\right)}{\theta_{\beta}} \mathrm{d} \theta_{\beta}\right)^{i},
$$

where $U_{i}$ can be calculated by solving equation (16) as $a$ varies. As $t$ increases, we calculate the approximations for $a=0, a=-0.0003, a=-0.005$, and $a=-0.03$ and the corresponding time by solving equation (32). The corresponding water profiles are shown in Figure 7 , where the thick solid line shows the steady solution in equation (6). The dotted lines show the present approximations, and solid lines show the results by the FEM as time increases. Compared with the FEM, the relative errors are shown in Figure 8.

The present approximation agrees with numerical solution, and the maximum relative error of present solution is less than $-3 \%$ in Figure 8. In addition, we solve equation (32) and derive the relationship between time variable $t$ and water content $\theta$, which is shown in Figure 9, and the relative errors are presented in Table 4.

Figure 7 The changes of water profile for the van Genuchten model. Dotted lines show the present approximations, and solid lines show the results by the FEM.

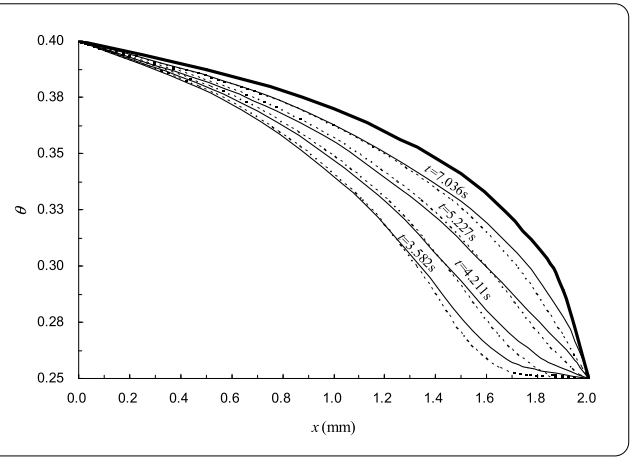

Figure 8 Relative errors of present approximations and the FEM.

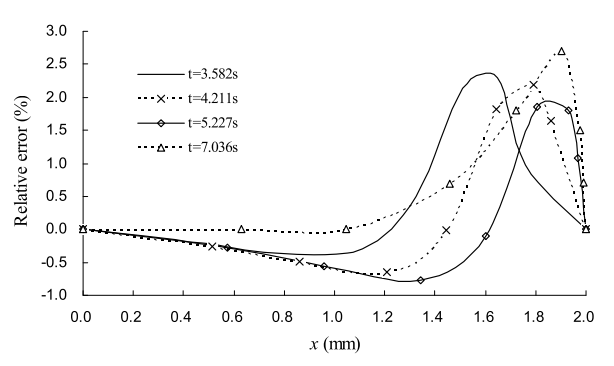


Figure 9 Relationship between $t$ and $\theta$ in $x_{d}=1.5 \mathrm{~mm}$.

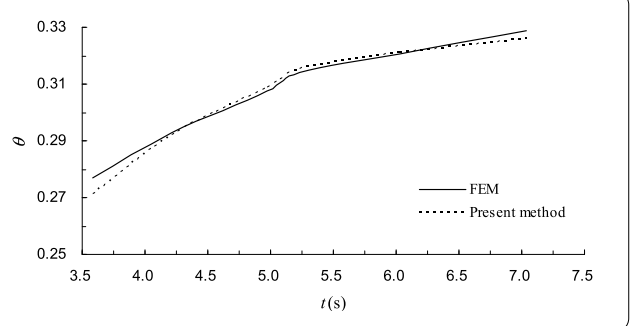

Table 4 The results of $\theta$ obtained from the present method and the FEM in $x_{d}=1.5 \mathrm{~mm}$

\begin{tabular}{llll}
\hline $\boldsymbol{t}(\mathbf{s})$ & Present method & FEM & Relative error (\%) \\
\hline 3.58233 & 0.27141 & 0.2771 & -2.05341 \\
4.211 & 0.29188 & 0.2929 & -0.34824 \\
4.97556 & 0.30889 & 0.3075 & 0.452033 \\
5.2274 & 0.31535 & 0.314 & 0.429936 \\
6.026 & 0.32109 & 0.3208 & 0.090399 \\
7.036 & 0.32607 & 0.32878 & -0.82426 \\
\hline
\end{tabular}

The present solution shown by dotted line approximates well with the FEM shown by solid lines in time simulation in Figure 9, and the maximum relative error is $-2.0534 \%$ in Table 4.

\section{Conclusion}

In this paper, we analyzed the changes of wetting front position and derived an approximate analytical solution of RE with finite boundaries (2). The solid lines in Figure 1 can be approximated by the solution equation (17), which is a series solution. According to equation (17), the series solution in fact is an approximation for semiinfinite boundary with different initial values, which means that the process of water infiltration varying from transient flow to steady one in Figure 1 can be simulated by the solution of semiinfinite problem with a variable initial value. The presented examples for the power law and van Genuchten model demonstrate the accuracy of the present solution by comparing the present results with the results obtained by the FEM.

Funding

The research has been supported by the Fundamental Research Funds for the Central Universities.

Competing interests

The authors declare that they have no competing interests.

Authors' contributions

Both authors contributed equally in this article. They read and approved the final manuscript.

Author details

${ }^{1}$ College of Civil Engineering, Tongji University, Shanghai, China. ${ }^{2}$ School of Aerospace Engineering and Applied

Mechanicas, Tongji University, Shanghai, China.

\section{Publisher's Note}

Springer Nature remains neutral with regard to jurisdictional claims in published maps and institutional affiliations.

Received: 14 June 2017 Accepted: 25 October 2017 Published online: 09 November 2017

\section{References}

1. Richards, LA: Capillary conduction of liquids through porous mediums. Physics 1, 318-333 (1931)

2. Witelski, TP: Perturbation analysis for wetting fronts in Richard's equation. Transp. Porous Media 27, $121-134$ (1997) 
3. Parlange, JY, Hogarth, WL, Parlange, MB, Haverkamp, R, Barry, DA, Ross, PJ, Steenhuis, TS: Approximate analytical solution of the nonlinear diffusion equation for arbitrary boundary conditions. Transp. Porous Media 30, 45-55 (1998)

4. Parlange, MB, Prasad, SN, Parlange, JY, Romkens, MJM: Extension of the Heaslet-Alksne technique to arbitrary soil water diffusivities. Water Resour. Res. 28, 2793-2797 (1992)

5. Witelski, TP: Motion of wetting fronts moving into partially pre-wet soil. Adv. Water Resour. 28, 1133-1141 (2005)

6. Parlange, JY, Hogarth, WL, Govindaraju, RS, Parlange, MB, Lockington, D: On an exact analytical solution of the Boussinesq equation. Transp. Porous Media 39, 339-345 (2000)

7. Omidvar, M, Barari, A, Momeni, M, Ganji, DD: New class of solutions for water infiltration problems in unsaturated soils. Geomech. Geoengin. 5, 127-135 (2010)

8. Olsen, JS, Telyakovskiy, AS: Polynomial approximate solutions of a generalized Boussinesq equation. Water Resour. Res. 49, 3049-3053 (2013)

9. Chen, X, Dai, Y: Differential transform method for solving Richards' equation. Appl. Math. Mech. 37, 169-180 (2016)

10. Tracy, FT: Clean two and three-dimensional analytical solution of Richards' equation for testing numerical solvers. Water Resour. Res. 42, 8503-8513 (2006)

11. Menziani, M, Pugnaghi, S, Vincenzi, S: Analytical solutions of the linearized Richards equation for discrete arbitrary initial and boundary conditions. J. Hydrol. 332, 214-225 (2007)

12. Hooshyar, M, Wang, DB: An analytical solution of Richards' equation providing the physical basis of SCS curve number method and its proportionality relationship. Water Resour. Res. 52, 6611-6620 (2016)

13. Galvis, J, Ki Kang, S: Spectral multiscale finite element for nonlinear flows in highly heterogeneous media: a reduced basis approach. J. Comput. Appl. Math. 260, 494-508 (2014)

14. Bougherara, B, Giacomoni, J: Existence of mild solutions for a singular parabolic equation and stabilization. Adv. Nonlinear Anal. 4, 123-134 (2015)

15. Pankavich, S, Michalowski, N: A short proof of increased parabolic regularity. Electron. J. Differ. Equ. 4, $521-531$ (2015)

16. Zaki, K, Redwane, H: Nonlinear parabolic equations with blowing-up coefficients with respect to the unknown and with soft measure data. Electron. J. Differ. Equ. 2016, Article ID 327 (2016)

17. Wang, HC, Liu, RK: The existence of stationary star solutions for compressible magnetohydrodynamic flows. Bound. Value Probl. 2016, $216(2016)$

18. $\mathrm{Ma}, \mathrm{TF}$, Yan, $\mathrm{BQ}$ : Positive global solutions of nonlocal boundary value problems for the nonlinear convection reaction-diffusion equations. Bound. Value Probl. 2017, 9 (2017)

19. Yang, J, Yu, HX: Sharp threshold for blow-up and global existence in a semilinear parabolic equation with variable source. Bound. Value Probl. 2017, 80 (2017)

20. Parlange, JY, Hogarth, WL, Fuentes, C, Sprintall, J, Haverkamp, R, Elrick, D, Parlange, MB, Braddock, RD, Lockington, DA: Superposition principle for short-term solutions of Richards equation - application to the interaction of wetting fronts with an impervious surface. Transp. Porous Media 17, 239-247 (1994)

21. Parlange, JY, Hogarth, WL, Fuentes, C, Sprintall, J, Haverkamp, R, Elrick, D, Parlange, MB, Braddock, RD, Lockington, DA: Interaction of wetting fronts with an impervious surface-longer time behavior. Transp. Porous Media 17, 249-256 (1994)

22. Guariglia, E: Fractional derivative of the Riemann zeta function. In: Cattani, C, Srivastava, HM, Yang, X-J (eds.) Fractional Dynamics, pp. 357-368. De Gruyter, Berlin (2015)

23. Lockington, D, Parlange, JY, Dux, P: Sorptivity and the estimation of water penetration into unsaturated concrete. Mater. Struct. 32, 342-347 (1999)

24. Hall, C: Water sorptivity of mortars and concretes: a review. Mag. Concr. Res. 41, 51-61 (1989)

25. Van Genuchten, MT: A closed-form equation for predicting the hydraulic conductivity of unsaturated soils. Soil Sci. Soc. Am. J. 44, 892-898 (1980)

26. Kolditz, O, Gorke, UJ, Shao, H, Wang, W: Thermo-Hydro-Mechanical-Chemical Processes in Porous Media, pp. 125-142. Springer, Berlin (2012)

27. Rucker, DF, Wrrick, AW, Ferre, TPA: Parameter equivalence for the Gardner and van Genuchten soil hydraulic conductivity functions for steady vertical flow with inclusions. Adv. Water Resour. 28, 689-699 (2005)

\section{Submit your manuscript to a SpringerOpen ${ }^{\circ}$ journal and benefit from:}

- Convenient online submission

- Rigorous peer review

- Open access: articles freely available online

- High visibility within the field

- Retaining the copyright to your article

Submit your next manuscript at $>$ springeropen.com 